\title{
Balıkesir Kıyı Kumullarındaki Bitki Çeşitliliği ve Tehdit Faktörleri
}

\author{
Fatih SATIL ${ }^{1}$, Gülendam TÜMEN², Selami SELVi ${ }^{*}$ \\ ${ }^{1}$ Balıkesir Üniversitesi, Fen-Edebiyat Fakültesi, Biyoloji Bölümü, Çağış Yerleşkesi, 10145, Balıkesir \\ ${ }^{2}$ TOKi Yenimahalle, Karesi/Balıkesir \\ ${ }^{3}$ Balıkesir Üniversitesi, Altınoluk Meslek Yüksekokulu, Tıbbi ve Aromatik Bitkiler Programı,10870, Balıkesir \\ Sorumlu yazar, e-posta: sselvi2000@yahoo.com \\ ORCID ID: https://orcid.org/0000-0002-9959-6945 \\ fsatil@gmail.com \\ gultumen@gmail.com \\ ORCID ID: $h$ ttps://orcid.org/0000-0002-4938-1161 \\ ORCID ID: https://orcid.org/0000-0002-7457-3341
}

Geliş Tarihi: 03.03.2021

Kabul Tarihi: 13.06.2021

Anahtar kelimeler

Balıkesir; Biyoçeşitlilik;

Ekoloji; Flora; Halofit; Kumul
Öz

Türkiye'nin 23 ilinde toplam 105 kıyı kumulu bulunmaktadır. Bu kumullarından 8'i Balıkesir ili sınırlarında yer almaktadır. Bu çalışma, 2016-2018 yıllarında Balıkesir' in Armutova, Çoruk, Belkıs Tombolosu ve Karabiga kıyı kumullarında gerçekleştirilmiştir. Elde edilen sonuçlar, daha önce çalışılan Ayvalık ve Gönen kumulları ile birlikte değerlendirilerek Balıkesir'deki 8 kumul alanın florası ortaya konmuştur. Floristik çalışmalar sonucunda alanda; 39 familyaya ait 120 cins ve 152 takson teşhis edilmiştir. Teşhisi yapılan türlerin 2'si Pteridophyta divizyosuna, 150' si ise Spermatophyta divizyosuna aittir. Spermatophyta divizyosuna ait taksonların tümü Angiospermae alt divizyosuna aittir. Angiospermae alt divizyosundan 134 tür Dicotyledonae, 16 tür ise Monocotyledonae sınıfına aittir. Tespit edilen bitki taksonlarından \%35'lik kısmı (54 takson) Akdeniz, \%4 (6 takson) Avrupa-Sibirya fitocoğrafik bölgesine ve \%61'lik (92 takson) kısmı da fitocoğrafik bölgesi tespit edilemeyen türlerden oluşmaktadır. Bölgedeki kumul alanların karakteristik florasında en çok takson içeren ilk üç familya; Asteraceae, Fabaceae ve Poaceae'dir. Kumul alanlarda en fazla taksona sahip olan cinsler, Plantago ve Limonium olup bunları Allium ve Juncus cinsleri takip etmektedir. Bütün araştırma alanlarımızda görülen en yaygın taksonlar ise Cakile maritima, Glaucium flavum ve Eryngium maritimum'dur. Çalışma alanında 1 endemik takson tespit edilmiştir. Endemik Trigonella coerulescens subsp. ayvalikensis'in doğal habitatı olan Sarımsaklı plajı turizm faaliyetleri nedeniyle yoğun insan baskısı altındadır. Ayrıca, kumul alanlarda görülen Pancratium maritimum'un doğal habitatlarından halk tarafından sökülmesi ve yayılış alanında plaj düzenlemesi gibi nedenlerle habitat bozulmaları türün popülasyonunu azaltmıştır. Bunların dışında, sanayi ve kanalizasyon sularının arıtılmadan denize akıtılması, kıyıya yakın tarımsal faaliyetler, çöp atma ve kıyılardan kum alımı gibi faaliyetler bölgedeki kumul habitatlar için önemli tehdit unsurlarıdır.

\section{Plant Diversity and Threat Factors in the Coastal Dunes of Balıkesir}

\begin{abstract}
There are a total of 105 dunes in 23 provinces of Turkey. 8 of these coastal dunes are located within the borders of Balıkesir province. This study was carried out in Armutova, Çoruk, Belkıs Tombolos and Karabiga coastal dunes located on the coasts of Balıkesir between 2016-2018. The obtained results were evaluated together with the Ayvalık and Gönen dunes previously studied, and the flora of 8 dune areas in Balıkesir was revealed. As a result of floristic studies; 120 genera and 152 taxa belonging to 39 families were identified. 2 of the species diagnosed are belonging to Pteridophyta diviso and 150 species belonging to Spermatophyta divisio. All of the taxa belonging to the Spermatophyta division belong to the Angiospermae subdivision. Of the Angiospermae subdivision, 134 species belong to the Dicotyledonae and 16 species belong to the Monocotyledonae class. Of the idendified plant taxa, 35\% (54 taxa) consists of Mediterranean, 4\% (6 taxa) to the European-Siberian phytogeographical region and $61 \%$ (92 taxa) of the species whose phytogeographical region can't be determined. The first three families that contain the most taxa in the characteristic flora of the dune areas in the region; Asteraceae, Fabaceae and Poaceae. The most common taxa in dune areas are belong to genera Plantago and Limonium, followed by Allium and Juncus. The most common taxa seen in all our research areas are Cakile maritima, Glaucium flavum, and Eryngium maritimum. One endemic taxon has been identified in the study area. Sarımsaklı beach, which is the natural habitat of the endemic taxon Trigonella coerulescens subsp. ayvalikensis, is under heavy human pressure due to tourism activities. In addition,
\end{abstract}


habitat degradation has decreased the population of the species due to reasons such as the removal of Pancratium maritimum from its natural habitats by the public and beach arrangement in the area of distribution. Apart from these, activities such as pouring industrial and sewage water into the sea without treatment, agricultural activities close to the shore, garbage disposal and taking sand from the coasts are important threats to the dune habitats in the region.

(C) Afyon Kocatepe Üniversitesi.

\section{Giriş}

Deniz ve kara ekosistemlerinin kesiştikleri geçiş bölgeleri olan kıyı ekosistemleri, biyoçeşitlilik ve deniz ile kara arasındaki dengeyi sağlaması bakımından önemli görevleri olan kumul alanlardır. Bu nedenle kıyılar, su ortamları ile karasal ortamlar arasında kendilerine özgü ekolojik özellikler taşıyan gerçek ekotonlar olarak bilinirler. Yeryüzünün sadece \%4'ünü meydana getiren kıyı alanları, dünya nüfusunun yaklaşık 1/3'ünü barındırmaktadır (Demiriz, 1969; Odum ve Barrett; 2008; $\mathrm{Avcl}$, 2017a,b,c). Kıyı kumulları bitki örtüsünün yetişme koşulları bakımından oldukça karmaşık habitatlardır. $\mathrm{Bu}$ alanların yüksek geçirgenliği ve bitki besin maddelerince fakir olması, doğrudan güneş ışığına maruz kalması, yüksek sıcaklık ve şiddetli rüzgâr gibi iklimsel faktörlere maruz kalması, yüzey hareketliliği ve denizden gelen ve yüksek oranda tuz içeren deniz suyuna maruz kalması gibi nedenler, kumullarda bitki örtüsünün gelişimini sınırlayan en önemli faktörlerdir.

Kumul alanlar, dünya üzerinde çok az ve dar alanlar olması nedeniyle doğa koruma açısından son derece değerli yaşam ortamları olarak kabul edilmektedirler. Ayrıca bu alanlar, yalnız kıyı şeridinde bulunan nadir ve nesli tükenme tehlikesi altındaki bitki ve hayvan türlerinin korunması açısından da hayati bir öneme sahiptirler (Özhatay ve Byfield, 1996).

Türkiye'nin 23 ilinde toplam 105 kıyı kumulu bulunmaktadır. Kıyı kumul florasının oldukça zengin olduğu ülkemizde kumulların toplam uzunluğu 8333 km'dir (Uslu 1988, Avcl vd. 2015). Erinç'e göre (2001); Türkiye'deki kumullarından $8^{\prime}$ i Balıkesir ili sınırlarında yer almaktadır. Balıkesir'deki bu kumulların üçü Ayvalık ilçesinde (Altınova, Sarımsaklı ve Badavut), ikisi Gönen'de (Gönen ve Karabiga deltaları), diğerleri ise Gömeç (Armutova), Edremit/Burhaniye (Çoruk) ve Erdek (Belkıs Tombolosu)'te yer alır. Bu kumullardan Ayvalık ve
Gönen floraları tarafımızdan incelenmiştir (Satıl vd. 2019a,b). Ancak Balıkesir'de yer alan Armutova, Çoruk, Belkıs Tombolosu ve Karabiga deltası kumul alanlarının florası ile ilgili bugüne kadar herhangi bir çalışma yapılmamıştır. Bunun dışında sadece bölgede Balıkesir-Izmir arasında kalan Edremit Körfezi-Gökova Körfezi arası alanın florası (Akyol ve Gemici 2017), Karaburun-Akdağ çevresinin flora ve vejetasyonu (Bekat 1980) ile Foça, Aliağa ve Çandarlı bölgesini vejetasyonu (Bekat ve Seçmen 1988) ile ilgili çalışmalar mevcuttur. Gökova ve Edremit Körfezleri arasında kalan Ege kıyılarının vejetasyon ekolojisini ve biyolojik çeşitliliğini çalışan Akyol ve Gemici (2017) alanda toplam 5 vejetasyon tipi ve 12 bitki birliği belirlemiştir. Ancak bu çalışmada saha ile ilgili bir floristik liste ortaya konmamıştır.

Bu çalışma ile Balıkesir ilinde daha önce çalışıımamış (Armutova, Çoruk, Belkıs Tombolosu ve Karabiga) 4 kıyı kumul alanının bitki çeşitliliği tespit edilmiştir. Ayrıca, daha önce çalışılan Ayvalık ve Gönen floraları ile de karşılaştırılmıştır. Böylece Balıkesir'in tüm kumul florası ortaya konması amaçlanmıştır.

\section{Materyal ve Metot}

Çalışma, 2016-2018 yılları arasında Balıkesir sınırlarındaki 4 kıyı kumul alanında (Armutova, Çoruk, Belkıs Tombolosu ve Karabiga deltası) gerçekleştirilmiştir (Şekil 1). Ayrıca çalışmada, daha önce ekibimizce çalışılan Ayvalık ve Gönen kumul florası verileri de kullanılmıştır (Satıl vd. 2019 a,b). 


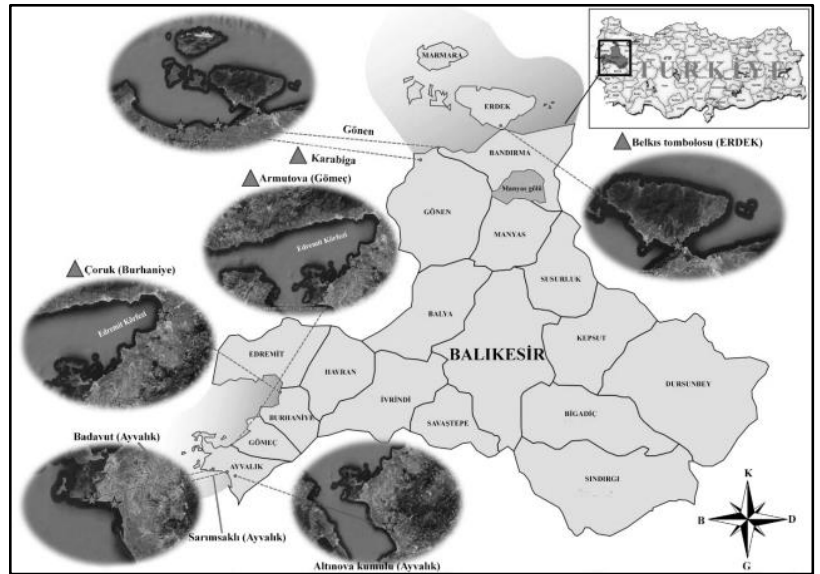

Şekil 1. Balıkesir kıyı kumul alanları (Bu çalışmada incelenen alanlar ( $($ ) ile gösterilmiştir.

Dört mevsim arazi çalışmaları sonucunda floristik ve ekolojik gözlemler yapılarak alanların bitki çeşitliliği tespit edilmiştir (Şekil 2).

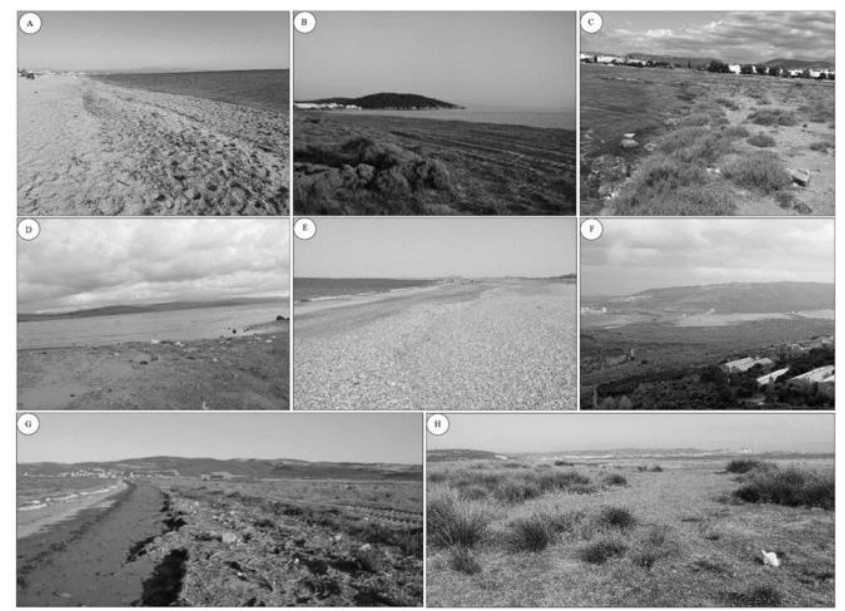

Şekil 2. Balıkesir kıyı kumullarından genel görüntüler. A: Sarımsaklı (Ayvalık), B: Badavut (Ayvalık), C: Altınova (Ayvalık), D: Armutova (Gömeç), E: Çoruk (Edremit/Burhaniye), F: Belkıs Tombolosu (Erdek), G: Gönen, H: Karabiga.

Floristik çalışmalar sonucunda, alandaki kumul ve tuzcul bitki taksonları toplanmış, alan fotoğrafları çekilmiş ve herbaryum materyalleri haline getirilmişlerdir. Tespit edilen bitki örnekleri GT 9601675 arasını kapsayan toplayıcı numarası ile Balıkesir Üniversitesi, Altınoluk Meslek Yüksekokulu Botanik Laboratuvarında muhafaza edilmektedir. Toplanan bitkilerin teşhisinde başta "Flora of Turkey" olmak üzere "Mountain Flora of Greece", "Flora of Europae" ve bölgede yapılan diğer floristik çalışmalardan yararlanılmıştır (Tutin vd. 1964-1980, Davis 1965-1985; Davis vd. 1988, Strid ve Tan 1991, Güner vd. 2000, Güner vd. 2012). Çalışmada tespit edilen taksonların Türkçe isimleri Güner vd. (2012)'a göre, güncel bilimsel isimleri Catalogue of Life'a (Roskov vd. 2019) ve taksonların otör adları Authors of Plants Name adlı eserle karşılaştırılmış ve otör isimleri kısaltılmıştır (Brummit ve Powel, 1992). Ayrıca taksonların tehlike kategorilerinin belirlenmesinde Ekim ve ark. (2000) ile IUCN (2019) den faydalanılmıştır.

\section{Bulgular}

Bu çalışmada Balıkesir ilinde yer alan Armutova, Erdek, Çoruk ve Karabiga kıyı kumulları floristik açıdan incelenmiştir. Ayrıca daha önce ekibimizce çalışılan Ayvalık ve Gönen kumul florası verileri de bu çalışmada kullanılarak böylece Balıkesir'deki tüm kumul alanların flora listesi karşılaştırmalı olarak ortaya konmuştur (Çizelge 1). Floristik çalışmalar sonucunda alanda; 39 familyaya ait 120 cins ve 152 takson tespit edilmiştir. Çizelge 1'de Balıkesir kumullarında yayılış gösteren bu taksonlar, yayılış gösterdiği kumul alanlar ve fitocoğrafik elementleri familya bazında alfabetik olarak verilmiştir. 
Çizelge 1. Balıkesir kıyı kumullarında tespit edilen taksonlar.

\begin{tabular}{|c|c|c|c|c|c|c|c|c|c|c|}
\hline No & Familya & Takson & Türkçe isim & $\begin{array}{c}{ }^{*} \text { Ayvalık }{ }^{1-} \\
3\end{array}$ & *Gönen & ${ }^{* *}$ Armutova & **Çoruk & **Belkıs & **Karabiga & $\begin{array}{c}\text { Fitocoğrafik } \\
\text { element }\end{array}$ \\
\hline 1 & Amaranthaceae & Amaranthus viridis $\mathrm{L}$. & Yeşilibik & + & - & +(GT1278) & + +(GT1165) & - & - & Bilinmiyor \\
\hline 2 & Amaranthaceae & Atriplex lasiantha Boiss. & Deliunluca & + & - & $+($ GT1300) & $+($ GT1111) & $+($ GT1102) & - & Bilinmiyor \\
\hline 3 & Amaranthaceae & Beta maritima L. var. maritima & Kıyıpancarı & + & + & $+($ GT1160) & $+($ GT1486) & + (GT1238) & $\boldsymbol{+}($ GT1100) & Bilinmiyor \\
\hline 5 & Amaranthaceae & Halimione portulacoides (L.) Aellen & Kocabetne & + & + & $+($ GT1200) & $+($ GT1175) & $+($ GT1476) & + (GT1113) & Bilinmiyor \\
\hline 6 & Amaranthaceae & Salicornia emericii Duval-Jouve & Denizbörülcesi & + & - & +(GT1211) & +(GT1191) & - & - & Bilinmiyor \\
\hline 7 & Amaranthaceae & Salsola kali L. & Döngele & + & + & +(GT1060) & +(GT1531) & $+(\mathrm{GT} 1170)$ & $+($ GT1212) & Bilinmiyor \\
\hline 8 & Amaranthaceae & Salsola soda $\mathrm{L}$. & Denizfasülyesi & + & + & $+($ GT1107) & $+($ GT1600) & $+($ GT1118) & $+($ GT1020) & Bilinmiyor \\
\hline 9 & Amaranthaceae & Suaeda prostrata Pall. & Yatıkcirim & + & + & $+($ GT1566) & +(GT960) & + (GT1086) & +(GT1661) & Bilinmiyor \\
\hline 10 & Amaranthaceae & Suaeda altissima Pall. & Cirimotu & - & - & - & - & $+($ GT1660) & $+($ GT1201) & Bilinmiyor \\
\hline 11 & Amaryllidaceae & Allium ampeloprasum L. & Pirasa & + & - & + (GT1103) & $\boldsymbol{+}(\mathrm{GT1675)}$ & $\boldsymbol{+}(\mathrm{GT} 1012)$ & - & Akdeniz \\
\hline 12 & Amaryllidaceae & Allium commutatum Guss. & İtdirseği & + & + & $+($ GT1031) & $+($ GT1000) & + (GT1243) & + (GT1466) & Akdeniz \\
\hline 13 & Amaryllidaceae & Allium roseum L. subsp. roseum & Gülsoğanı & + & + & $+($ GT1642) & $+($ GT1112) & $+($ GT1180) & + (GT1150) & Akdeniz \\
\hline 14 & Amaryllidaceae & Allium sphaerocephalon L. & Yılansarmısağ & + & - & $+($ GT1375) & - & $+($ GT1550) & - & Avrupa-Sibirya \\
\hline 15 & Amaryllidaceae & Narcissus tazetta L. subsp. tazetta & Nergis & + & + & +(GT1087) & +(GT961) & - & - & Bilinmiyor \\
\hline 16 & Amaryllidaceae & Pancratium maritimum L. & Kumzambağı & + & + & $+($ GT1641) & + (GT1598) & $+($ GT1237) & $+($ GT1307) & Akdeniz \\
\hline 17 & Anacardiaceae & Pistacia lentiscus L. & Sakızağacı & + & - & - & - & - & - & Akdeniz \\
\hline 18 & Apiaceae & Bunium ferulaceum Sibth. \& Sm. & İncirop & - & + & - & - & - & +(GT1565) & Akdeniz \\
\hline 19 & Apiaceae & Cachrys cristata DC. & Tepeliçarşambaotu & - & + & - & - & $+($ GT1530) & +(GT1630) & Akdeniz \\
\hline 21 & Apiaceae & Eryngium maritimum L. & Kumboğadikeni & + & + & $+($ GT1121) & $+($ GT1567) & $+($ GT1244) & +(GT1011) & Bilinmiyor \\
\hline 22 & Apiaceae & Ferula communis L. subsp. communis & atkasnağl & - & - & - & - & + (GT1456) & $\boldsymbol{+}($ GT1385) & Akdeniz \\
\hline 23 & Apiaceae & Oenanthe pimpinelloides L. & Delimaydanoz & - & + & - & - & - & $\boldsymbol{+}($ GT1239) & Bilinmiyor \\
\hline 24 & Apocynaceae & Cynanchum acutum L. subsp. acutum & Bacirgan & + & + & $+($ GT1181) & $+($ GT1117) & $+($ GT1185) & + (GT1123) & Bilinmiyor \\
\hline 25 & Apocynaceae & Cionura erecta (L.) Griseb. & Babrik & - & + & - & - & $+($ GT1084) & - & Bilinmiyor \\
\hline 26 & Araceae & Arum italicum Mill. & Domuzlahanası & - & + & +(GT1132) & $+($ GT1122) & +(GT1213) & - & Bilinmiyor \\
\hline 27 & Asparagaceae & Ornithogalum pyrenaicum L. & Eșeksusamı & - & + & - & $+($ GT1190) & +(GT1370) & +(GT1145) & Bilinmiyor \\
\hline 28 & Asparagaceae & Prospero autumnale (L.) Speta & Güzsümbülü & - & + & + (GT1195) & $+($ GT1297) & +(GT1021) & +(GT1532) & Akdeniz \\
\hline 29 & Asteraceae & Achillea maritima (L.) Ehrend. \& Y.P.Guo & Çocukotu & + & - & - & - & $+($ GT1128) & $+($ GT1549) & Akdeniz \\
\hline 30 & Asteraceae & Anthemis rigida (Sibth. \& Sm.) Boiss. & Diripapatya & + & - & +(GT1088) & $+($ GT1133) & +(GT1597) & +(GT1568) & Akdeniz \\
\hline 31 & Asteraceae & Anthemis tomentosa L. subsp. tomentosa & Sahilpapatyası & + & - & $+($ GT1236) & + (GT1083) & $+($ GT1242) & - & Akdeniz \\
\hline 32 & Asteraceae & Artemisia santonicum L. subsp. santonicum & Denizyavşanı & + & + & $+($ GT1601) & $+($ GT1564) & + (GT1658) & +(GT1059) & Bilinmiyor \\
\hline 33 & Asteraceae & Asteriscus aquaticus (L.) Less. & Saritop & - & + & $+($ GT1032) & $+($ GT1131) & $+($ GT1013) & $+($ GT1631) & Akdeniz \\
\hline 34 & Asteraceae & Bellis annua $\mathrm{L}$. & Akbubeçlik & + & + & $+($ GT1640) & $+($ GT1061) & $+($ GT1241) & $+($ GT1663) & Akdeniz \\
\hline 35 & Asteraceae & Cardopatium corymbosum (L.) Pers. & Kurtludiken & + & + & $+($ GT1202) & $+(\mathrm{GT} 1620)$ & +(GT1533) & +(GT1602) & Akdeniz \\
\hline 36 & Asteraceae & Carduus acicularis Bertol. & Sivrikangal & - & + & - & - & - & + (GT1529) & Akdeniz \\
\hline 37 & Asteraceae & Carlina gummifera (L.) Less. & Sakızkeyganası & - & + & - & - & $+($ GT1287) & $+($ GT1548) & Akdeniz \\
\hline 38 & Asteraceae & Carthamus caeruleus L. & Gökaspir & + & - & - & - & - & - & Bilinmiyor \\
\hline 39 & Asteraceae & Centaurea spinosa L. var. spinosa & Denizgeveni & - & + & - & - & + (GT1082) & $+($ GT1142) & Bilinmiyor \\
\hline
\end{tabular}


Asteraceae Cota tinctoria (L.) J.Gay var. tinctoria

Asteraceae

Asteraceae

Asteraceae

Asteraceae

Asteraceae

Asteraceae

Asteraceae

Asteraceae

Asteraceae

Asteraceae

Boraginaceae

Brassicaceae

Brassicaceae

Brassicaceae

Brassicaceae

Brassicaceae

Brassicaceae

Brassicaceae

Brassicaceae

Campanulaceae

Caryophyllaceae

Caryophyllaceae

Caryophyllaceae

Caryophyllaceae

Caryophyllaceae

Caryophyllaceae

Caryophyllaceae

Caryophyllaceae

Cistaceae

Convolvulaceae

Convolvulaceae

Crassulaceae

Cucurbitaceae

Cyperaceae

Cyperaceae

Cyperaceae

Cyperaceae

Cyperaceae

Equisetaceae

Euphorbiaceae

Euphorbiaceae

Fabaceae

Fabaceae

Fabaceae
Crepis commutata (Spreng) Greuter

Hedypnois rhagadioloides (L.) F.W.Schmidt

Helichrysum orientale (L.) Gaertn.

Helminthotheca echioides (L.) Holub

Hypochaeris achyrophorus $\mathrm{L}$.

Hypochaeris glabra $\mathrm{L}$.

Inula graveolens (L.) Desf.

Senecio vernalis Waldst. \& Kit.

Tripolium pannonicum (Jacq.) Dobrocz

Xanthium strumarium L. subsp. strumarium

Echium angustifolium Mill.

Alyssum umbellatum Desv.

Cakile maritima Scop.

Eruca vesicaria (L.) Cav.

Malcolmia flexuosa (Sibth. \& Sm.) Sibth.

Matthiola sinuata (L.) R.Br.

Matthiola tricuspidata (L.) R.Br.

Raphanus raphanistrum $\mathrm{L}$.

Rapistrum rugosum (L.) All.

Jasione montana L. subsp. montana

Arenaria serpyllifolia L.

Paronychia echinulata Chater

Sagina maritima G.Don

Silene behen $\mathrm{L}$

Silene gallica $\mathrm{L}$.

Spergula arvensis $\mathrm{L}$.

Spergularia marina (L.) Besser

Silene fabaria Sibth. \& Sm.

Tuberaria guttata (L.) Fourr.

Calystegia soldanella (L.) R.Br.

Cressa cretica L.

Sedum litoreum Guss. var. litoreum

Bryonia alba $\mathrm{L}$.

Bolboschoenus maritimus (L.) Palla

Carex muricata L. subsp. muricata

Cyperus capitatus Vand.

Eleocharis palustris (L.) Roem. \& Schult.

Scirpoides holoschoenus (L.) Soják

Equisetum telmateia Ehrh.

Euphorbia paralias L.

Euphorbia peplis L.

Hippocrepis unisiliquosa $\mathrm{L}$.

Hymenocarpos circinnatus (L.) Savi

Lotus corniculatus L. var. corniculatus
Boyacıpapatyası

Delikıskis

Sünnetlice

Sarısolmaz

Billurdüğme

Sarıhindiba

Cıbıldağmarulu

Delisarıot

Kanaryaotu

Bataklıkpapatyası

Kocapitrak

Agres

Şișkinkevke

Kumteresi

Roka

Kayaşebboyu

Oyalışebboy

Öküzșebboyu

Eşekturpu

Kediturpu

Dağgökçesi

Kurukumotu

Kıyıkepekotu

Arsizsaginotu

Akkıvşak

Serçeçiçeği

Tarlakişnişi

Sahilremilotu

Kösenakıl

Karagözgülü

Kumsarmașı̆

Reçineçiçeği

Kiyıkoruğu

Akhaylin

Sandalyesazı

Cengelsazı

Sehvetotu

Delisaz

Vurla

Deredoruk

Kumsütleğeni

Kıyısütleğeni

Atnalı

Pulluot

Gazalboynuzu

\begin{tabular}{|c|c|c|c|c|}
\hline +(GT1141) & +(GT1446) & +(GT1390) & +(GT1569) & Bilinmiyor \\
\hline +(GT1062) & +(GT1619) & +(GT1081) & +(GT1365) & Bilinmiyor \\
\hline +(GT1240) & +(GT1563) & +(GT1528) & +(GT1325) & Akdeniz \\
\hline- & - & +(GT1534) & +(GT1547) & Bilinmiyor \\
\hline +(GT1089) & +(GT1022) & +(GT1603) & +(GT1596) & Bilinmiyor \\
\hline - & +(GT1235) & +(GT1349) & $\boldsymbol{+}$ (GT1140) & Akdeniz \\
\hline - & - & - & - & Akdeniz \\
\hline - & - & +(GT1049) & +(GT1300) & Akdeniz \\
\hline +(GT1436) & +(GT1151) & +(GT1546) & +(GT1326) & Bilinmiyor \\
\hline - & - & +(GT1204) & +(GT1058) & Avrupa-Sibir \\
\hline +(GT1143) & +(GT963) & +(GT1080) & +(GT1277) & Bilinmiyor \\
\hline +(GT1215) & +(GT1570) & +(GT1148) & +(GT1535) & Akdeniz \\
\hline +(GT1127) & +(GT1360) & +(GT1161) & +(GT1618) & Akdeniz \\
\hline +(GT1033) & +(GT1426) & +(GT1014) & +(GT964) & Bilinmiyor \\
\hline +(GT1643) & +(GT1193) & +(GT1090) & +(GT1023) & Bilinmiyor \\
\hline +(GT1395) & - & - & - & Akdeniz \\
\hline +(GT1079) & +(GT1063) & +(GT1324) & $\boldsymbol{+}_{\text {(GT1632) }}$ & Bilinmiyor \\
\hline $\boldsymbol{+}$ (GT1496) & + (GT1158) & +(GT1015) & +(GT1664) & Akdeniz \\
\hline +(GT1571) & +(GT1527) & +(GT1245) & + (GT1135) & Bilinmiyor \\
\hline +(GT1048) & +(GT1216) & +(GT1562) & +(GT1526) & Bilinmiyor \\
\hline - & - & +(GT1595) & - & Bilinmiyor \\
\hline - & - & +(GT1617) & +(GT1171) & Bilinmiyor \\
\hline - & - & +(GT1348) & - & Akdeniz \\
\hline +(GT1400) & +(GT1323) & +(GT1572) & +(GT1057) & Bilinmiyor \\
\hline +(GT1153) & +(GT1301) & +(GT1657) & +(GT1350) & Bilinmiyor \\
\hline +(GT1205) & +(GT1233) & +(GT1016) & +(GT1267) & Bilinmiyor \\
\hline $\boldsymbol{+}$ (GT1416) & +(GT1545) & +(GT1064) & +(GT1024) & Bilinmiyor \\
\hline +(GT1034) & +(GT1506) & +(GT1536) & +(GT1665) & Bilinmiyor \\
\hline +(GT1234) & +(GT978) & +(GT1246) & +(GT1633) & Bilinmiyor \\
\hline- & - & +(GT1405) & +(GT1065) & Bilinmiyor \\
\hline +(GT1604) & +(GT1001) & +(GT1217) & +(GT1525) & Bilinmiyor \\
\hline +(GT1137) & +(GT965) & - & - & Bilinmiyor \\
\hline +(GT1174) & +(GT1035) & +(GT1163) & +(GT1347) & Akdeniz \\
\hline- & - & - & +(GT1516) & Avrupa-Sibiry \\
\hline +(GT1302) & +(GT1066) & +(GT1168) & +(GT1130) & Bilinmiyor \\
\hline +(GT1078) & +(GT1047) & +(GT1594) & - & Avrupa-Sibiry \\
\hline+ (GT1616) & +(GT1147) & +(GT1206) & - & Bilinmiyor \\
\hline $\boldsymbol{+}$ (GT1639) & +(GT1003) & +(GT1257) & - & Bilinmiyor \\
\hline $\boldsymbol{+}$ (GT1593) & $\boldsymbol{+}(\mathrm{GT1355})$ & $\boldsymbol{+}$ (GT1544) & - & Bilinmiyor \\
\hline - & - & + (GT1178) & + (GT1327) & Bilinmiyor \\
\hline +(GT1573) & $+($ GT1002) & +(GT1250) & +(GT1537) & Akdeniz \\
\hline +(GT1077) & +(GT1674) & +(GT1656) & +(GT1056) & Akdeniz \\
\hline+ (GT1339) & +(GT1605) & +(GT1251) & - & Bilinmiyor \\
\hline +(GT1561) & +(GT1303) & +(GT1524) & - & Bilinmiyor \\
\hline +(GT977) & +(GT1346) & +(GT1410) & - & Bilinmiyor \\
\hline
\end{tabular}


Fabaceae

Fabaceae

Fabaceae

Fabaceae

Fabaceae

Fabaceae

Fabaceae

Fabaceae

Fabaceae

Fabaceae

Fabaceae

Fabaceae

Frankeniaceae

Gentianaceae

Geraniaceae

Geraniaceae

Juncaceae

uncaceae

Juncaceae

Juncaceae

Lamiaceae

Lythraceae

Papaveraceae

Papaveraceae

Papaveraceae

Plantaginaceae

Plantaginaceae

Plantaginaceae

Plantaginaceae

Plantaginaceae

Plumbaginaceae

Plumbaginaceae

Plumbaginaceae

Plumbaginaceae

Plumbaginaceae

Poaceae

Poaceae

Poaceae

Poaceae

Poaceae

Poaceae

Poaceae

Poaceae

30 Poaceae
Lotus ornithopodioides L.

Lupinus angustifolius L. subsp.

Lupinus angustifolius L. subsp. angustifolius

Medicago marina L.

Medicago orbicularis (L.) Bartal.

Onobrychis caput-galli (L.) Lam.

Ornithopus compressus L.

Scorpiurus subvillosus L. var. subvillosus L

Trifolium repens $\mathrm{L}$. var. repens

Trifolium subterraneum

Trigonella coerulescens (M.Bieb.) Halácsy subsp. ayvalikensis Erdoğan Selvi\& Tümen (E)

Vicia villosa Roth. subsp. eriocarpa P.W.Ball

Frankenia hirsuta L.

Centaurium maritimum (L.) Fritsch

Erodium acaule (L.) Becherer \& Thell.

Geranium molle L.

Juncus acutus L. subsp. acutus

Juncus bufonius $\mathrm{L}$.

Juncus capitatus Weigel

Juncus gerardi Loisel. subsp. gerardi

Vitex agnus-castus L.

Lythrum tribracteatum Salzm. ex Ten.

Glaucium flavum Crantz

Hypecoum procumbens $\mathrm{L}$

Papaver rhoeas $\mathrm{L}$

Plantago afra L.

Plantago bellardii All.

Plantago coronopus $\mathrm{L}$

Plantago lagopus $\mathrm{L}$.

Plantago lanceolata $\mathrm{L}$

Limonium angustifolium (Tausch) Turrill

Limonium bellidifolium (Gouan) Dumort

Limonium graecum (Poir.) Rech.f.

Limonium sinuatum (L.) Mill.

Limonium virgatum (Willd.) Fourr.

Aegilops biuncialis Vis.

Aeluropus littoralis (Gouan) Parl.

Arundo donax L

Brachypodium distachyon (L.) P.Beauv.

Cynodon dactylon (L.) Pers. var. villosus Rege

Echinochloa crus-galli (L.) P. Beauv.

Elymus farctus (Viv.) Runemark ex Melderis

E. flaccidifolius (Boiss. \& Heldr.) Melderis

Holcus annuus Salzm. ex C.A.Meyer
Civcivayağ

Yahudibaklası

Acıbakla

Sahilyoncası

Paralık

Pitrakkorunga

Kuşayağ

Koyundücüğü

Aküçgül

Yeraltıüçülü

Ayvalıkçemen

Boğala

Tülpembe

Sarıgelindüğmesi

Leylekgagası

Yumuşakitır

Kofa

Kamır

Topakkofa

Erkekkofa

Hayıt

Üçaklarotu

Gündürmelâlesi

Düğmecik

Gelincik

Ateșyaprağı

Babadeşen

Clı̆nak

Kırkdamarotu

Damarlica

Sahilkaranfili

Hoskuduzotu

Kumkaranfil

Denizlavantas

Cilızot

Íkikılçı

Sahilayrı̆̆

Kargl

Tekkılcan

Köpekdiși

Darıcan

Cicora

Bataklıkcicorası

Giygin

\begin{tabular}{|c|c|c|c|c|}
\hline+ (GT1615) & +(GT1157) & +(GT1218) & - & Akdeniz \\
\hline +(GT966) & +(GT1574) & - & - & Bilinmiyor \\
\hline +(GT1036) & +(GT1188) & +(GT1207) & +(GT1328) & Bilinmiyor \\
\hline +(GT1523) & +(GT1232) & +(GT1025) & +(GT1345) & Bilinmiyor \\
\hline$+($ GT1076) & +(GT1345) & +(GT1046) & +(GT1543) & Bilinmiyor \\
\hline$+($ GT1322) & +(GT1247) & +(GT1538) & +(GT1125) & Akdeniz \\
\hline+ (GT1338) & +(GT1592) & - & - & Akdeniz \\
\hline+ (GT1167) & +(GT1575) & +(GT1173) & +(GT1231) & Bilinmiyor \\
\hline +(GT1542) & +(GT1304) & +(GT1198) & +(GT1055) & Bilinmiyor \\
\hline$+($ GT1184) & +(GT1208) & +(GT1606) & +(GT1340) & Bilinmiyor \\
\hline - & - & - & - & Bilinmiyor \\
\hline+ (GT1067) & +(GT1183) & +(GT1415) & - & Bilinmiyor \\
\hline - & - & - & - & Bilinmiyor \\
\hline+ (GT1321) & +(GT1219) & +(GT1539) & +(GT1591) & Akdeniz \\
\hline+ (GT1560) & +(GT1177) & +(GT1305) & +(GT1329) & Akdeniz \\
\hline +(GT1614) & +(GT1522) & +(GT1655) & +(GT1634) & Bilinmiyor \\
\hline +(GT1037) & +(GT1344) & +(GT1335) & +(GT1068) & Bilinmiyor \\
\hline +(GT1644) & +(GT1208) & +(GT1220) & +(GT1026) & Bilinmiyor \\
\hline +(GT967) & +(GT1673) & +(GT1252) & +(GT1576) & Bilinmiyor \\
\hline+ (GT1646) & +(GT1004) & +(GT1613) & +(GT1666) & Bilinmiyor \\
\hline+ (GT1194) & +(GT1330) & +(GT1017) & +(GT1045) & Akdeniz \\
\hline +(GT1541) & + (GT1450) & +(GT1253) & +(GT1607) & Bilinmiyor \\
\hline +(GT976) & +(GT1362) & +(GT1069) & +(GT1120) & Bilinmiyor \\
\hline$+($ GT1075) & +(GT1104) & +(GT1237) & +(GT1635) & Bilinmiyor \\
\hline+ (GT1645) & +(GT1451) & +(GT1209) & +(GT1306) & Bilinmiyor \\
\hline +(GT1577) & +(GT1187) & +(GT1204) & +(GT1054) & Bilinmiyor \\
\hline+ (GT1343) & + (GT1452) & +(GT1540) & - & Akdeniz \\
\hline+ (GT1218) & +(GT1038) & +(GT1189) & +(GT1010) & Akdeniz \\
\hline$+($ GT1590) & +(GT1672) & + +(GT968) & +(GT1638) & Akdeniz \\
\hline +(GT975) & +(GT1152) & +(GT1654) & +(GT1009) & Bilinmiyor \\
\hline +(GT1608) & $+($ GT1330) & +(GT1230) & +(GT1667) & Akdeniz \\
\hline - & - & - & - & Avrupa-Sibirya \\
\hline - & - & +(GT1521) & +(GT1307) & Akdeniz \\
\hline +(GT1320) & +(GT1364) & - & - & Akdeniz \\
\hline +(GT1337) & +(GT1227) & +(GT1254) & +(GT1008) & Akdeniz \\
\hline +(GT1091) & +(GT1612) & +(GT1018) & +(GT1027) & Bilinmiyor \\
\hline +(GT1095) & +(GT1363) & +(GT1361) & +(GT1114) & Bilinmiyor \\
\hline +(GT1197) & +(GT1341) & +(GT1256) & +(GT1668) & Bilinmiyor \\
\hline +(GT1050) & $+($ GT1070) & +(GT1019) & +(GT1044) & Akdeniz \\
\hline +(GT1096) & +(GT1005) & +(GT1051) & +(GT1342) & Bilinmiyor \\
\hline +(GT1647) & +(GT1039) & +(GT1578) & +(GT1115) & Bilinmiyor \\
\hline +(GT1094) & +(GT1210) & - & - & Bilinmiyor \\
\hline +(GT1331) & +(GT1609) & +(GT1028) & +(GT974) & Bilinmiyor \\
\hline +(GT1097) & +(GT1309) & +(GT1228) & +(GT969) & Akdeniz \\
\hline
\end{tabular}

512 


\begin{tabular}{|c|c|c|c|c|c|c|c|c|c|c|}
\hline 131 & Poaceae & Phragmites australis (Cav.) Trin. ex Steudel & Kamış & + & - & + (GT1648) & $\boldsymbol{+ ( \mathrm { GT } 1 0 3 0 )}$ & +(GT1255) & + (GT1007) & Avrupa-Sibirya \\
\hline 132 & Poaceae & Poa bulbosa L. & Yumrulusalkım & + & - & $+($ GT1092) & $+($ GT1670) & $+($ GT1332) & $+($ GT1636) & Bilinmiyor \\
\hline 133 & Poaceae & Stipa capensis Thunb. & Kumkılaçı & + & + & $+(\mathrm{GT} 1040)$ & +(GT1162) & +(GT1124) & + (GT1172) & Akdeniz \\
\hline 134 & Poaceae & Elymus elongatus (Host) Runemark & Putaotu & - & - & - & - & $\boldsymbol{+}($ GT1653) & $+($ GT1520) & Bilinmiyor \\
\hline 135 & Polygonaceae & Polygonum maritimum L. & Sicimlik & + & + & +(GT1071) & $+(\mathrm{GT} 1134)$ & +(GT1308) & +(GT1579) & Bilinmiyor \\
\hline 136 & Polygonaceae & Rumex pulcher L. subsp. pulcher & Ekşilik & + & - & $+($ GT1611) & $+(\mathrm{GT} 1226)$ & $\boldsymbol{+}($ GT1652) & $+($ GT1053) & Bilinmiyor \\
\hline 137 & Primulaceae & Anagallis arvensis $\mathrm{L}$. & Farekulağı & + & - & $+($ GT1098) & - & - & - & Bilinmiyor \\
\hline 138 & Primulaceae & Anagallis arvensis L. var. arvensis & Farekulağı & + & - & +(GT970) & $+($ GT1325) & $+($ GT1333) & $+($ GT1110) & Bilinmiyor \\
\hline 139 & Rosaceae & Sarcopoterium spinosum (L.) Spach & Abdestbozan & + & + & - & - & - & - & Akdeniz \\
\hline 140 & Rubiaceae & Crucianella imbricata Boiss. & Kurakhaçotu & + & - & +(GT1258) & $+(\mathrm{GT} 1144)$ & - & - & Akdeniz \\
\hline 142 & Rubiaceae & Galium brevifolium Sm. & Sünnetotu & + & - & - & - & - & - & Akdeniz \\
\hline 143 & Rubiaceae & Galium caminianum Schult. & Bursasünnetlicesi & - & + & - & - & - & +(GT973) & Akdeniz \\
\hline 144 & Rubiaceae & Sherardia arvensis L. & Gökörenotu & + & - & $+($ GT1580) & +(GT1041) & $\boldsymbol{+}($ GT1154) & $+($ GT1637) & Akdeniz \\
\hline 145 & Rubiaceae & Valantia hispida $\mathrm{L}$. & Kıllıörenotu & + & + & $+($ GT1217) & +(GT1182) & + (GT1029) & + (GT1479) & Akdeniz \\
\hline 146 & Rubiaceae & Valantia muralis $\mathrm{L}$. & Örenotu & + & - & $+($ GT1099) & +(GT1669) & $+($ GT1224) & +(GT1610) & Akdeniz \\
\hline 147 & Santalaceae & Thesium humile Vahl. & Bodurgüvelek & + & + & $+($ GT1478) & $+($ GT1006) & $\boldsymbol{+}($ GT1073) & $+($ GT1334) & Akdeniz \\
\hline 148 & Selaginellaceae & Selaginella denticulata (L.) Link & Dişliselagin & + & - & $+($ GT1259) & $+(\mathrm{GT} 1222)$ & $+($ GT1336) & $+($ GT1074) & Bilinmiyor \\
\hline 149 & Tamaricaceae & Tamarix tetrandra Pall. ex Bieb. & Gezik & + & + & $+($ GT1093) & $+($ GT1207) & $+($ GT1651) & $+($ GT1105) & Bilinmiyor \\
\hline 150 & Typhaceae & Typha angustifolia L. & Saz & + & - & $+($ GT1650) & + (GT1335) & +(GT971) & +(GT1164) & Bilinmiyor \\
\hline
\end{tabular}

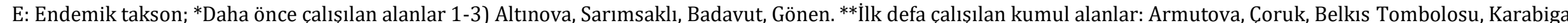




\section{Tartışma ve Sonuç}

Floristik çalışmalar sonucunda Balıkesir'deki 8 kıyı kumul alanında (Ayvalık, Altınova, Sarımsaklı, Badavut, Armutova, Çoruk, Belkıs Tombolosu ve Karabiga); 39 familyaya ait 120 cins ve 152 taksonun yayılış gösterdiği ortaya konmuştur. Bu kumul alanlarından; Altınova, Sarımsaklı, Badavut ve Gönen kumullarındaki bitki çeşitliliği Satıl vd (2019 a,b) tarafından tespit edilmiş olup bu çalışmamızda Balıkesir ilindeki tüm kumul alanlardaki bitki çeşitliliği toplu halde verilmiştir. Alanda sadece bir endemik taksona (Trigonella coerulescens subsp. ayvalikensis) rastlanmış olup endemizm oranı \% 0.7 olarak belirlenmiştir. Alanda yapılan floristik çalışmalar sonucunda en fazla taksona sahip olan familya Asteraceae (24 takson) olup; bunu sırasıyla Fabaceae (15 takson), Poaceae (13 takson) ve Amaranthaceae (10 takson) takip etmektedir (Şekil 3). Çalışma alanımıza yakın olan Çanakkale'de önemli kumul ve tuzcul alanların florası ve ekolojisi üzerine yapılan bir çalışmada ise bizim bulgulardan farklı olarak; toplam 51 familya, 164 cins ve 257 takson tespit edilmiş, bizim bulgulara benzer şekilde ilk üç familyanın Poaceae, Asteraceae ve Fabaceae olduğu görülmüştür (Özmen ve Uysal 2012). Çalışma alanlarımızdan Burhaniye ve Ayvalık kumullarına yakın Edremit körfezi kumul florası üzerine çalışma yapan Güçlü (2019); alanda 56 familya ve 180 cinse ait toplam 238 takson tespit etmiştir. En çok cins içeren familyalar Asteraceae (25), Poaceae (19) ve Fabaceae (17) olmasıyla bizim sonuçlarla benzerlik göstermektedir. Gönen, Karabiga ve Erdek kumullarına benzer iklim ve karaktere sahip Mudanya sahilinde Daşkın (2012) tarafından yapılan floristik bir çalışmada da 55 familya, 181 cinse ait toplam 252 takson tespit edilmiştir. En zengin familyalar; Asteraceae (42), Poaceae (33), Chenopodiaceae/Caryophyllaceae (12)'dir. Bu çalışma sonuçlarına göre taksonların familyalara göre sıralanması az bir farkla bizim çalışma ile benzerlik göstermektedir.

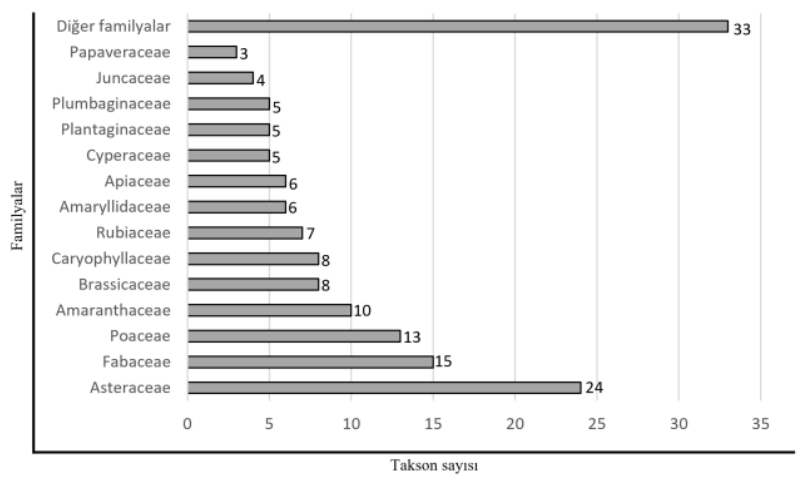

Şekil 3. Balıkesir kıyı kumullarında tespit edilen taksonların familya bazında dağıımı.

Flora of Turkey'e göre en çok cins içeren familyalar Poaceae, Asteraceae, Apiaceae, Brassicaceae, Fabaceae olarak sıralanmaktadır (Davis 1965-1985, Davis vd. 1988). Sıralama açısından küçük farklar bulunmasına rağmen bizim çalışmamızdaki familya sıralaması ile büyük ölçüde benzerlik göstermektedir. Ayrıca, Asteraceae, Fabaceae ve Poaceae familyalarının çalışma alanında en fazla tür içeren ilk üç familya olması, içerdikleri takson sayısı fazlalığı ve farklı yaşam ortamlarına uyum sağlama özellikleri ile açıklanabilir. Bunlara ek olarak, kıyı kumulları üzerinde oluşan antropojenik baskı ve zarar göz önüne alındığında bu familya üyelerinin daha yaygın görülmesi doğal karşılanmaktadır. Taksonların cins bazında dağılımları incelendiğinde; en fazla taksona sahip olan cinslerin $5^{\prime}$ er taksonla Plantago ve Limonium olduğu, bunları 4'er taksonla Allium ve Juncus cinslerinin takip ettiği görülmektedir (Şekil 4).

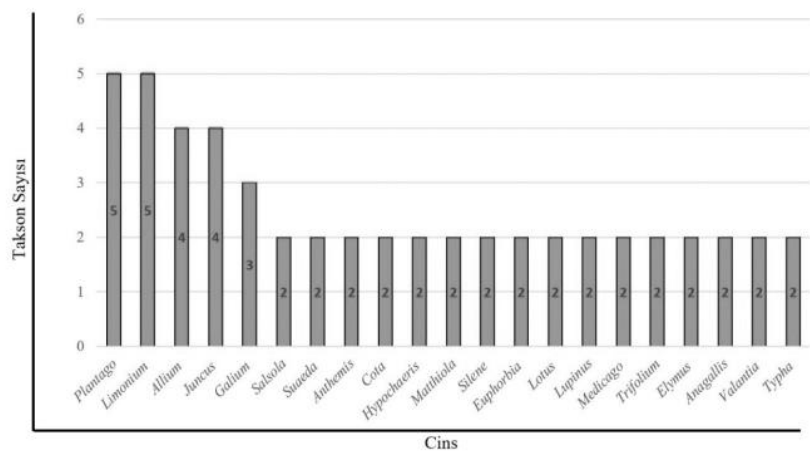

Şekil 4. Balıkesir kıyı kumullarında tespit edilen taksonların cins bazında dağııımı 
Taksonların fitocoğrafik bölgelerine dağılışı incelendiğinde; Akdeniz Elementi \%35 (54 takson), Avrupa-Sibirya Elementi \%4 (6 takson) ile bilinmeyenler \%61 (92 takson) şeklindedir (Şekil 5). Güçlü (2019) tarafından Edremit körfezi kumul florası üzerine yapılan bir çalışmada tespit edilen taksonların fitocoğrafik bölgere göre dağılımı; 91 Akdeniz (\%37.91), 12 Avrupa-Sibirya (\%5) ve 2 tür ve tür altı takson İran-Turan (\%0.83) olarak belirlenmiştir. Bölgesi bilinmeyen ya da birden fazla bölgeye dahil olan takson sayısı ise 132'dir (\%56)'dır. $\mathrm{Bu}$ veriler bizim çalışma verileri ile benzerlik göstermektedir. Gönen, Karabiga ve Belkıs Tombolosu kumullarına benzer iklim ve karaktere sahip Mudanya kıyı florasında da taksonların fitocoğrafik bölgelere göre dağılımı; 55(\%22)'i Akdeniz, 17(\%6.8)'si Avrupa-Sibirya, 3 (\%1.2)'ü İranTuran ve $175(\% 70)^{\prime} i$ de çok bölgeli ya da bölgesi bilinmeyenler şeklindedir (Daşkın 2012). Sonuç olarak Mudanya florasının sonuçları da bizim sonuçlara paralellik göstermektedir.

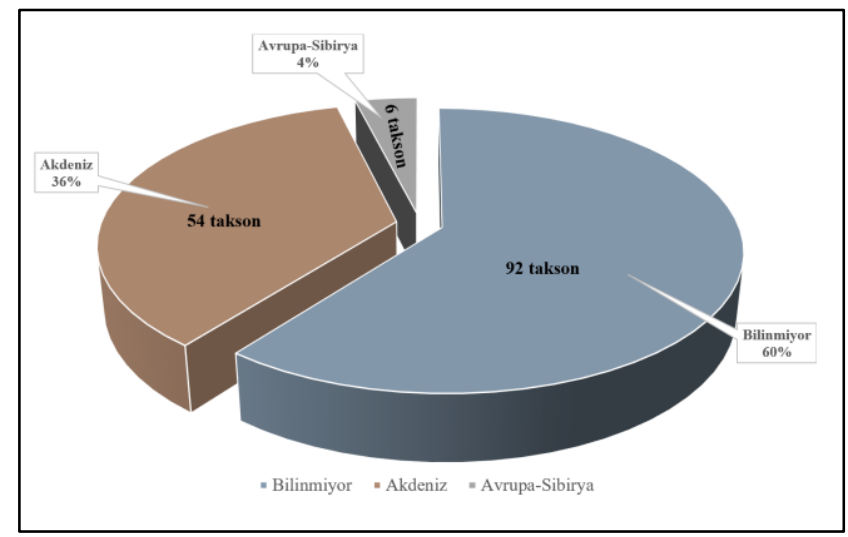

Şekil 5. Balıkesir kıyı kumullarında tespit edilen taksonların fitocoğrafik elementlere göre dağılımı

Bu çalışmada ayrıca Balıkesir'deki 8 kumul alanın florası kendi içinde karşılaştırılmıştır (Şekil 6). Buna göre; Gönen ilçesindeki kumul florasının 127 taksonla (\%83.55) en zengin kumul alanı olduğu görülmektedir. Gönen kumullarını 120 taksonla (\%78.95) Karabiga kumulu takip etmektedir (Şekil 6). Takson sayısının diğer kumullara nazaran Gönen ve Karabiga da yüksek olması, söz konusu bu alanların coğrafik konumu ve iklim çeşitliliğine bağlı olarak hem Akdeniz hem de Öksin elementlerin birlikte bulunması ile açıklanabilir. Ayrıca, Çizelge 2'de, araştırma sonuçlarımız ile araştırma alanımıza yakın olan bölgelerde yapılan floristik çalışmalar karşılaştırılmıştır. Çizelge 2'de de görüleceği gibi çalışma alanlarımızdan yakın bölgede yapılan tüm çalışmalarda en çok takson sayısına sahip familya sıralaması ve fitocoğrafik elementler açısından benzerlik görülmektedir. Edremit ve Ayvalık körfez bölgelerindeki kumullarının takson sayıları birbirine yakın görülmektedir. Çalışılan kumullar arasında en az takson sayısına Belkıs Tombolosu (Erdek)'nda rastlanmıştır. Bunun nedeni şöyle açıklanabilir; Belkıs Tombolosu, alüvyal düzlük, kum ve çakıllardan meydana gelmiştir. Bu tombolosun doğusunda kumullar, batısında plaj kumları, kuzey ve güneyinde alüvyal alanlar, ortasında ise bir bataklık yer almaktadır. Yaklaşık $1 \mathrm{~km}$ uzunluğundaki bu bataklık alan genellikle kuzeyden gelen iki akarsuyun taşıdığı malzeme ile sürekli olarak daralmaktadır (Cürebal vd. 1998). Aktif kumul sahasının kuzeybatı kenarında kalan kumullar üzerinde DSi tarafından yerleştirilen sazlıklarla; bu sahadaki kumul hareketi önlenmeye çalışılmıştır (Hapçıoğlu, 1977).

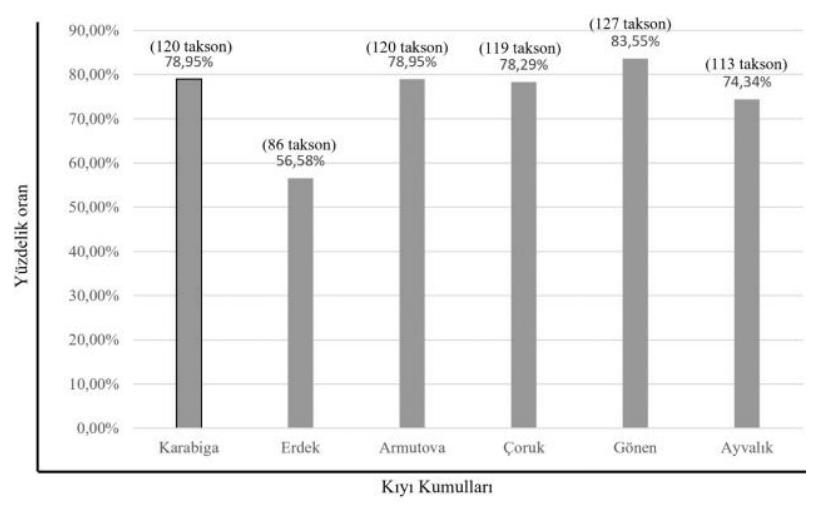

Şekil 6. Balıkesir kıyı kumullarında tespit edilen toplam takson sayısının kumul alanlarına göre yüzdelik dağılımları 
Çizelge 2. Çalışma alanları ile yakın çevredeki benzer çalışmaların karşılaştııılması

\begin{tabular}{|c|c|c|c|c|c|c|}
\hline & Çalışma Alanı & Familya & Cins & Tür & Baskın Fitocoğrafik Dağılım & Baskın İlk Üç Familya \\
\hline \multirow{20}{*}{ 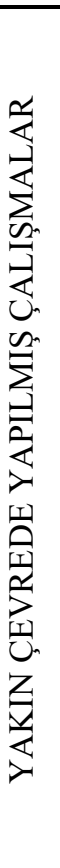 } & Edremit Körfezi & 56 & 180 & 238 & $\% 37.91$ Akdeniz Elementi & Asteraceae (32 takson) \\
\hline & Güçlü (2019) & & & & & Fabaceae (28 takson) \\
\hline & & & & & & Poaceae (23 takson) \\
\hline & Çanakkale & 51 & 164 & 257 & $\% 35$ Akdeniz Elementi & Poaceae (43 takson) \\
\hline & Özmen ve Uysal (2012) & & & & & Asteraceae (33 takson) \\
\hline & & & & & & Fabaceae (29 takson) \\
\hline & Armutlu Yarımadası & 52 & & 212 & - & Lamiaceae (20 takson) \\
\hline & İnan (1994) & & & & & Asteraceae (20 takson) \\
\hline & & & & & & Fabaceae (19 takson) \\
\hline & Mudanya & 55 & 181 & 252 & $\% 22$ Akdeniz Elementi & Asteraceae (42 takson) \\
\hline & Daşkın (2012) & & & & & Poaceae (33 takson) \\
\hline & & & & & & Chenopodiaceae (12 takson) \\
\hline & Armutlu Yarımadası & 37 & 153 & 312 & \%9.5 Akdeniz Elementi & Asteraceae (123 takson) \\
\hline & Kaynak (1997) & & & & & $\begin{array}{l}\text { Fabaceae ( } 91 \text { takson) } \\
\text { Brassicaceae ( } 41 \text { takson) }\end{array}$ \\
\hline & Ayvalık & 33 & 91 & 118 & $\% 36$ Akdeniz Elementi & Asteraceae (16 takson) \\
\hline & Satıl vd. (2019) & & & & & Fabaceae (15 takson) \\
\hline & & & & & & Poaceae (8 takson) \\
\hline & Gönen & 32 & 75 & 86 & $\% 20$ Akdeniz Elementi & Asteraceae (17 takson) \\
\hline & Sat1l vd. (2019) & & & & & Poaceae (7 takson) \\
\hline & & & & & & Brascicae (7 takson) \\
\hline \multirow{11}{*}{ 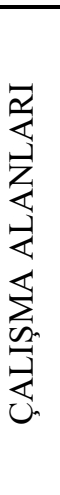 } & Karabiga (Gönen) & 33 & 93 & 150 & $\% 35$ Akdeniz Elementi & Asteraceae (19 takson) \\
\hline & & & & & & Poaceae (12 takson) \\
\hline & & & & & & Brassicaceae (7 takson) \\
\hline & Belkıs Tombolosu (Erdek) & 18 & 33 & 42 & $\% 33$ Akdeniz Elementi & Asteraceae (10 takson) \\
\hline & & & & & & $\begin{array}{l}\text { Amaryylidaceae (4 takson) } \\
\text { Poaceae ( } 3 \text { takson) }\end{array}$ \\
\hline & Çoruk (Burhaniye) & 32 & 93 & 117 & $\% 32$ Akdeniz Elementi & Asteraceae (14 takson) \\
\hline & & & & & & Fabaceae (14 takson) \\
\hline & & & & & & Poaceae (12 takson) \\
\hline & Armutova (Gömeç) & 32 & 92 & 117 & $\% 32$ Akdeniz Elementi & Fabaceae (14 takson) \\
\hline & & & & & & Asteraceae (13 takson) \\
\hline & & & & & & Poaceae (12 takson) \\
\hline
\end{tabular}


Balıkesir'deki kumul alanları tehdit eden faktörler:

Çalışma alanımızdaki kıyı kumul alanlarının hepsi turizm sahası içerisinde yer almaktadır. Bu nedenle tüm kumul alan habitatları, ikinci konut inşaatı ve plaj düzenlemeleri gibi faaliyetler nedeniyle yoğun baskı altındadır. Avcı (2017c), Türkiye'de kumul alanların, en fazla yerleşmeler tarafından tehdit edildiğini ifade etmektedir. Çalışılan tüm kumul alanlarda ortak sorun olan ikinci konutların önce Marmara denizinin kuzey kıyılarından başlayarak güneye doğru yayılmasıyla Erdek, Edremit, Burhaniye ve Ayvalık gibi yerlerde turizm alanları gelişmiş. Nüfusun kumullar üzerindeki olumsuz etkisi zaman içinde artarak devam etmiştir. Sarımsaklı kumullarında lokal endemik $T$. coerulescens subsp. ayvalikensis plajlar bölgesinde yayılış göstermesi nedeniyle doğal habitatları turizm baskısı altındadır (Şekil 7a). Ayrıca, bölgedeki kumul alanlarda Pancratium maritimum soğanlarının halk tarafından toplanıyor olması ve yayılış alanında plaj düzenlemeleri nedeniyle habitat bozulmaları bu türün popülasyonlarını oldukça düşürmüştür. Bu amaçla, P. maritimum populasyonları üzerinde Milli Parklar Şube Müdürlüğü ve yerel yönetim işbirliği ile koruma çalışmaları başlatmışlardır. Ancak bu çalışmalar yeterli düzeyde değildir (Şekil $7 b, c)$. Hem $T$. coerulescens subsp. ayvalikensis hem de $P$. maritimum taksonları ile ilgili tür eylem planlarına intiyaç vardır.

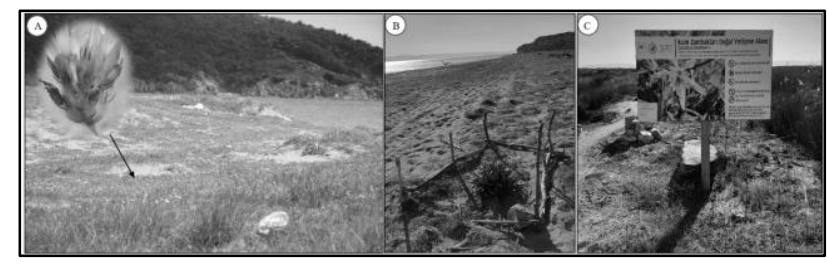

Şekil 7. Ayvalık kıyı kumullarında; A) T. coerulescens subsp. ayvalikensis habitatı, B-C) P. maritimum türünü koruma çalışmaları ve alınan önlemler.

Karabiga Beldesi çevresinde yapılan termik santrallerin oluşturduğu habitat tahribatı, kıyı ve karasal ekosistem üzerinde kirliliği neden olmaktadır. Erduran Nemutlu ve Doğan (2020), Karabiga deltası civarında, sanayi ve turizm alanlarının bölge değerleri dikkate alınmaksızın planlamalar yapıldığı ifade etmektedir. Çalışmada yaptığımız gözlemlerde söz konusu alanda yanlış yer seçimi sonucu kumul alan florası ve biyolojik çeşitliliği zarar gördüğü tespit edilmiştir (Şekil 8a). Kıraç ve Veryeri (2016), Karabiga ve çevresi ile ilgili hazırladıkları bir raporda Karabiga kıyıları, Kocabaş Çayı ve Kocabaş Deltası'nın uluslararası ölçütlere göre değerlendirildiğinde Önemli Doğa Alanı (ÖDA) olacak kalitede bir alan olduklarını belirtmektedir. Ancak, söz konusu kıyı şeridi son zamanlarda çok ciddi bir habitat tahribatı tehdidi altındadır. Bu bölgedeki kumul alanların korunabilmesi için, kıyı bölgesini tahrip eden sanayi alanları, ekolojik faktörler gözetilerek yapılacak yeni planlamalar ile farklı bölgelere taşınmalıdır.

Bölgedeki kumul alanları tehdit eden diğer sorunlar; kirlilik, çöp atma, denizden kum alımı ve plaj düzenlemeleri sonucu kumul alan tahribatıdır. Erdek, Edremit, Burhaniye ve Ayvalık gibi yerlerde plaj düzenlemeleri, Gönen, Belkıs Tombolosu ve Karabiga'da kirlilik ile birlikte kıyıya çöp atılması ve tarımsal faaliyetler kumul alanları ve florayı tehdit eden faktörlerdir (Şekil 8).

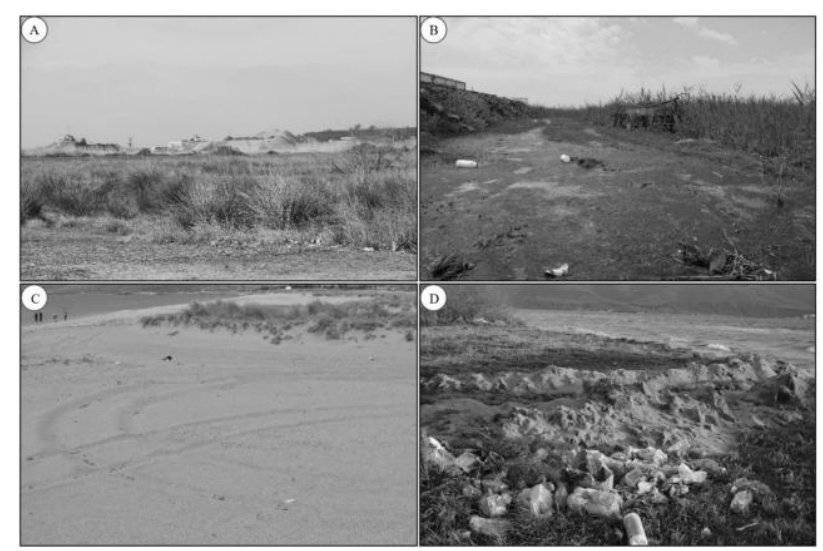

Şekil 8. Çalışılan kıyı kumullarında görülen çevresel sorunlar (A: Karabiga, kum alımı; B: Gönen, kirlilik; C: Çoruk/Armutova, plaj düzenlemesi; D: Belkıs Tombolosu, çöp yığınları) 


\section{Kaynaklar}

Akyol, Y. ve Gemici, Y., 2017. Kıyı Ege'nin (Gökova ve Edremit Körfezleri Arası) Vejetasyon Ekolojisi ve Biyolojik Çeşitliliğinin Ekolojik Yönetimi. Kastamonu Üniversitesi, Orman Fakültesi Dergisi, 17 (1), 116-123.

Avcl, M., Avcl, S. ve Akkurt, S. 2015. Coastal dune vegetation in Turkey: a geographical perspective. International Conference on the Mediterranean Coastal Environment MEDCOAST 2015, Varna, Bulgaristan, 6-10 Ekim 2015, vol.1: 397-405.

Avcı, M., 2017a, Türkiye'nin Kıyı Kumullarında Bitki Örtüsü, Yasal ve Bilimsel Boyutlarıyla: Kıyı (Ed. H. Turoğlu ve H. Yiğitbaşıoğlu), Jeomorfoloji Derneği Yayını No: 1, Anka matbaa, İstanbul: 63-92.

Avcı, M., 2017b. Türkiye'nin Kıyı Kumulları, Bitki Örtüsü ve Önemi. International Symposium on Geomorphology, 12-14 October 2017, Elazığ/Türkiye.

Avcı, S., 2017c, Kıyı Alanların Kullanımında Beşeri Faktörler. Yasal Bilimsel Boyutlarıyla Kıyı, Turoğlu, H., Yiğitbaşıŏlu (Editörler), Jeomorfoloji Derneği, İstanbul, 117-146.

Bekat, L., 1980. Karaburun-Akdağ çevresinin flora ve vejetasyonu, Yüksek Lisans tezi, Ege Üniversitesi Fen Bilimleri Enstitüsü, İzmir, 34.

Bekat, L. and Seçmen, Ö., 1988. Vegatation In der umgebung von Foça, Aliağa und Çandarlı. Journal of Faculty of Science Ege University Series B, 10 (2), 1527.

Brummit, R.K. and Powel, C.E., 1992, Authors of Plant Names, Royal Botanic Gardens, Kew, 732.

Cürebal, i., Kızılçaoğlu, A. ve Soykan, A., 1998. Belkıs Tombolosunun Jeomorfolojik ve Uygulamalı Jeomorfolojik Özellikleri. Balıkesir Üniversitesi Sosyal Bilimler Enstitüsü Dergisi, 1(1), 1-23.

Daşkın, R. 2012. Vascular Plants of Mudanya Cost (Bursa, South Marmara/Turkey). Biological Diversity and Conservation, 5(3), 28-36.

Davis, P., 1965-1985, Flora of Turkey and East Aegean Islands, Vol. 1-9, Edinburgh University Press, Edinburgh.
Davis, P., Mill, R.R. and Tan, K., 1988, Flora of Turkey and East Aegean Islands (Supplements I), Vol. 10, Edinburgh University Press, Edinburgh.

Demiriz, H., 1969. Armutlu (Gemlik) Çevresinin Florası Hakkında (Zur Flora von Armutlu bei Gemlik). Acta Biologica Turcica, 19 (2-4), 107-109.

Ekim T., Koyuncu M., Vural M., Duman H., Aytaç Z. ve Adıgüzel N., 2000, Türkiye Bitkileri Kırmızı Kitabı. Eğrelti ve Tohumlu Bitkiler. Ankara: Barışcan Ofset, 196.

Erduran Nemutlu, F. ve Doğan, Z., 2020. Karabiga (Çanakkale) Beldesi Mevcut Alan Kullanımlarının Belirlenmesi. ÇOMÜ Ziraat Fakültesi Dergisi, 8 (1), 107-113.

Erinç, S., 2001, Jeomorfoloji II (3.Basım), Der Yayınları No: 294, İstanbul.

Güçlü, H., 2019. Edremit körfezi (Balıkesir-Çanakkale) kumul florası, Yüksek Lisans Tezi, Gazi Üniversitesi Fen Bilimleri Enstitüsü Biyoloji Ana Bilim Dalı, Ankara, 100.

Güner, A., Aslan, S., Ekim, T., Vural, M., ve Babaç, M.T. (edlr.), 2012, Türkiye Bitkileri Listesi (Damarlı Bitkiler). Nezahat Gökyiğit Botanik Bahçesi ve Flora Araştırmaları Derneği Yayınları, İstanbul.

Güner, A., Özhatay, N., Ekim, T., and Başer, K.H.C., 2000, Flora of Turkey and East Aegean Islands (Supplements II), Vol. 11, Edinburgh University Press, Edinburgh.

Hapçıoğlu, N., 1977. Kapıdağ Kıyılarında Jeomorfolojik Gözlemler. iü Coğrafya Enstitü Dergisi,: 22, 203-210.

IUCN, 2019. The IUCN Red List of Threatened Species. Version. 20192. http://www.iucnredlist.org. Downloaded on 18 July 2019

İnan, M., 1994. Armutlu yarımadasının florası. Yüksek Lisans Tezi, İstanbul Üniversitesi Fen Bilimleri Enstitüsü, İstanbul, 54

Kaynak, G., 1997. Flora of Armutlu Peninsula III, Lagascalia 20(1), 63-98.

Kıraç, C.O ve Veryeri, N.O., 2016, Güneybatı Marmara Denizi ve Karabiga Kıyılarında (Biga, Çanakkale) 
Biyolojik Çeşitlilik ve Deniz-Kıyı Habitatları Araştırma

Sonuç Raporu. SAD Yayını. Ankara. 42.

Odum, E.P. ve Barrett, G.W., 2008, Ekolojinin Temel İlkeleri (Editör K. Işık). Ankara, Palme Yayıncılık.

Özhatay, N. ve Byfield, A., 1996, Türkiye'nin Kuzey Kumullarının Korunmasına Yönelik Rapor, Doğal Hayatı Koruma Derneği, İstanbul.

Özmen H.ve Uysal, í. 2012. Çanakkale' de (Türkiye) Önemli Kumul ve Tuzcul Alanların Florası ve Ekolojisi, 21. Ulusal Biyoloji Kongresi, 03-07 Eylül 2012, Ege Üniversitesi, İzmir, 669-670.

Roskov, Y., Ower, G., Orrell, T., Nicolson, D., Bailly, N., Kirk, P.M., Bourgoin, T., DeWalt, R.E., Decock, W., Nieukerken, E. van, Zarucchi J., Penev, L., eds. 2019, Species 2000 \& ITIS Catalogue of Life, 2019 Annual Checklist. Digital resource at www.catalogueoflife.org/annualchecklist/2019.Speci es 2000: Naturalis, Leiden, the Netherlands. ISSN 2405-884X.

Satıl, F., Tümen, G. ve Selvi, S., 2019a. Ayvalık (Balıkesir/Turkey) Dune Plant Diversity, Threatening Factors and Solution Proposals. Journal of the Institute of Science and Technology, 9(3), 1289-1298.

Satıl, F., Tümen, G. ve Selvi, S. 2019b. Gönen deltası kumul bitki çeşitliliği, tehdit faktörleri ve çözüm önerileri. Artvin Çoruh Üniversitesi Orman Fakültesi Dergisi, 20(2), 208-217.

Strid, A. ve Tan, K. (eds.) 1991, Mountain flora of Greece. Vol. 2., Edinburgh University Press. Edinburgh, UK.

Tutin, T.G., Heywood, V.H., Burges, N.A., Valentine, D.H., Walters, S.M. and Webb, D.A., 1964-1980, Flora Europae, Vol: 1-5, Cambridge at Univ. Press, Cambridge.

Uslu, T., 1988. Türkiye Kıyı Kumulları. IX. Ulusal Biyoloji Kongresi, 21-23 Eylül 1988, Sivas. 175- 183. 